\title{
CORRELAÇÃO ENTRE DIGESTIBILIDADE E COMPORTAMENTO INGESTIVO DE NOVILHAS SUPLEMENTADAS A PASTO\#
}

\author{
CORRELATION BETWEEN DIGESTIBILITY AND INGESTIVE BEHAVIOR OF HEIFERS \\ SUPPLEMENTED AT PASTURE
}

Santana Júnior, H.A. ${ }^{1}$, Silva, R.R. ${ }^{1 *}$, Carvalho, G.G.P. ${ }^{2}$, Silva, F.F. ${ }^{1}$, Mendes, F.B.L. ${ }^{1}$, Abreu Filho, G. ${ }^{1}$, Trindade Júnior, G. ${ }^{1}$, Cardoso, E.O. ${ }^{1}$, Barroso, D.S. ${ }^{1}$ e Pereira, M.M. ${ }^{1}$

1Universidade Estadual do Sudoeste da Bahia. Itapetinga-BA. Brasil. *rrsilva.uesb@hotmail.com ${ }^{2}$ Universidade Federal da Bahia. Itapetinga-BA. Brasil.

\author{
PaLAVRAS CHAVE ADICIONAIS \\ Bocado. Bovino. Digestão. Pastejo.
}

\section{RESUMO}

Objetivou-se, com este estudo, avaliar as correlações entre a digestibilidade aparente e o comportamento ingestivo de novilhas mestiças a pasto. O experimento foi conduzido na fazenda Princesa do Mateiro, município de Ribeirão do Largo, Bahia. Foram utilizadas 20 novilhas com grau de sangue 5/8 Guzerá linhagem leiteira e 3/ 8 Holandesa, com média de 18 meses de idade e peso corporal médio de $187 \pm 13,1 \mathrm{~kg}$. O experimento teve duração de 224 dias e os animais criados em pastejo rotacionado de Brachiaria brizantha 'Marandú'. Verificou-se uma correlação positiva entre o tempo de pastejo e o coeficiente de digestibilidade da fibra em detergente neutro (CDFDN). Correlações negativas foram verificadas entre o tempo de outras atividades e CDFDN. O tempo de alimentação no cocho apresentou correlações negativas com coeficiente de digestibilidade da matéria seca (CDMS), matéria orgânica (CDMO), carboidratos não fibrosos (CDCNF), carboidratos totais (CDCHOT). Observou-se uma correlação positiva entre o tempo de alimentação total e mastigação total com o CDFDN. O número de períodos de pastejo e ruminação apresentaram correlações negativas com CDMS, CDMO, CDCNF, CDCHOT. Também, verificou-se correlação acima de 0,90 para os nutrientes digestíveis totais (NDT), podendo também ser incluída em equações de predição de

"Parte da dissertação do primeiro autor e projeto financiado pelo Banco do Nordeste do Brasil-BNB.

\author{
Additional KEYWORDS \\ Mouthful. Cattle. Digestion. Grazing.
}

consumo. O número de período de alimentação no cocho apresentou correlação negativa com CDMO e coeficiente de digestibilidade de proteína bruta (CDPB). O tempo por período de ruminação apresentou correlação positiva com CDMO, CDCNF, CDCHOT, NDT e CDPB. O tempo por período de outras atividades apresentou correlação positiva com CDCNF e NDT. Correlações negativas foram verificadas entre a taxa de bocados e CDMS, CDMO, CDCNF, CDCHOT e CDPB. O tempo por deglutição correlacionou positivamente com CDMS, CDMO, CDCNF, CDCHOT, NDT, CDPB. A massa do bocado e bocados por deglutição não apresentaram correlações com nenhuma das variáveis da digestibilidade. O número de mastigação merícica por dia apresentou correlações negativas com NDT. O tempo de pastejo e ruminação não apresentaram relação com os demais coeficientes de digestibilidade aparente de novilhas mestiças a pasto. $O$ coeficiente da digestibilidade da fibra em detergente neutro provoca variações no tempo de pastejo.

\section{SUMMARY}

The objective of this study was to evaluate the correlation between the apparent digestibility and ingestive behavior of crossbred heifers in grass. The experiment was conducted at Princesa do Mateiro farm, Ribeirao do Largo, Bahia. Were used 20 heifers with blood level $5 / 8$ Guzerá milk lineage and $3 / 8$ Holstein, with an average of 18 months of 


\section{SANTANA JÚNIOR ETAL.}

age and body weight of $187 \pm 13.1 \mathrm{~kg}$. The experiment lasted 224 days and the animals raised in a rotational grazing on Brachiaria brizantha 'Marandu'. It was a positive correlation between grazing time and digestibility of neutral detergent fiber (CDFDN). Negative correlations were found between the time from other activities and CDFDN. Feeding time at trough showed negative correlations with digestibility of dry matter (CDMS), organic matter (CDMO), non-fibrous carbohydrates (CDCNF), total carbohydrates (CDCHOT). A positive correlation between total time spent eating and chewing with the total CDFDN was observed. The number of periods of grazing and rumination showed negative correlations with CDMS, CDMO, CDCNF, CDCHOT. Also, there was a correlation above 0.90 to the total digestible nutrients (TDN) and may also be included in intake prediction equations. The number of feeding periods at trough showed a negative correlation with CDMO and digestibility of crude protein (CDPB). The time spent for rumination was positively correlated with CDMO, CDCNF, CDCHOT, NDT and CDPB. The time spent for other activities positively correlated with CDCNF and TDN. Negative correlations were found between bite rate and CDMS, CDMO, CDCNF, CDCHOT and CDPB. Time swallowing positively correlated with CDMS, CDMO, CDCNF, CDCHOT, NDT, CDPB. The mass of the bit and mastications for swallowing did not correlate with any variable of digestibility. The number of ruminating chews per day correlated negatively with TDN. Grazing and ruminating times did not show any other relation with apparent digestibility of crossbred heifers on pasture. The coefficient of digestibility of neutral detergent fiber causes changes in grazing time.

\section{INTRODUÇÃO}

Os bovinos em pastejo têm a capacidade de adaptação às mais diversa condições de oferta de pasto, manejo e ambiente, modificando seus parâmetros de comportamento alimentar para alcançar e manter determinado nível de consumo compatível com as exigências nutricionais, o qual depende de outras variáveis, como a qualidade das forragens, sobretudo os teores de fibra, que está associada ao estímulo da mastigação, produção de saliva, motilidade do rúmen e manutenção ruminal (Cardoso et al., 2006).

O número de estudos de comportamento ingestivo de ruminantes tem elevado consideravelmente devido a sua relevância na interpretação dos efeitos encontrados nas pesquisas. Entretanto, associações entre variáveis comportamentais e metabólicas têm sido encontradas nas discussões dos trabalhos, na maioria das vezes, sem o devido embasamento científico dessas relações.

A digestibilidade do alimento é a capacidade do animal de aproveitar seus nutrientes em maior ou menor escala, expressa pelo coeficiente de digestibilidade dos nutrientes em apreço, e é uma característica influenciada tanto pelo animal como pelo alimento (Silva e Leão, 1979). Segundo Van Soest (1994), a digestibilidade pode ser definida como um processo de conversão de macromoléculas dos nutrientes em compostos mais simples, que podem ser absorvidos no trato gastrintestinal. As medidas de digestibilidade servem para qualificar o alimento quanto ao seu valor nutritivo e indicam o percentual de cada nutriente do alimento que o animal potencialmente pode aproveitar.

A digestibilidade aparente de um alimento é considerada a proporção do ingerido que não foi excretada nas fezes, não considerando a matéria metabólica fecal (MMF), representada principalmente pelas secreções endógenas, contaminação por microorganismos e descamações do epitélio (Berchielli et al., 2006). Segundo Minson (1990), a quantidade de MMF secretada nas fezes varia de 0,098 a 0,129 g/g de matéria seca ingerida.

Objetivou-se, com este estudo, avaliar as correlações entre a digestibilidade aparente e o comportamento ingestivo de novilhas mestiças a pasto.

\section{MATERIALE MÉTODOS}

O experimento foi conduzido na fazenda Princesa do Mateiro, município de Ribeirão 


\section{CORRELAÇÕES ENTRE DIGESTIBILIDADE E COMPORTAMENTO}

do Largo, localizado na região sudoeste do estado da Bahia. Foram utilizadas 20 novilhas mestiças com grau de sangue $5 / 8$ Guzerá linhagem leiteira e 3/8 Holandesa, com média de 18 meses de idade e peso corporal médio inicial de $187 \pm 13,1 \mathrm{~kg}$. O experimento teve duração de 224 dias, tendo seu início em 25 de maio de 2008 e finalizando no dia 04 de janeiro de 2009, sendo composto por quatro períodos de 56 dias.

Os animais criados em pastejo rotacionado de Brachiaria brizantha 'Marandú' em área de 10 ha, divididos em quatro piquetes de áreas equivalentes, sendo que os mesmos foram vedados 30 dias antes do início do experimento.

Foi fornecida suplementação concentrada diariamente (tabela I), às 10:00 horas, em cochos plásticos, coletivos com duplo acesso, sem cobertura, com dimensionamento linear de $80 \mathrm{~cm} /$ animal.

Os animais foram identificados nas unidades experimentais por meio de suas características morfológicas e brincos plásticos numerados.

As amostras da forragem do pastejo

Tabela I. Proporções de ingredientes do concentrado, na base da matéria natural. (Proportions, \% on fresh matter of concentrated ingredients).

\begin{tabular}{|c|c|c|c|c|}
\hline & \multicolumn{4}{|c|}{ Períodos } \\
\hline & $1 \stackrel{\circ}{0}$ & $2^{\circ}$ & 3 & $4 \stackrel{\circ}{-}$ \\
\hline Milho grão moído & 28,20 & 66,47 & 70,52 & 72,05 \\
\hline Farelo de soja & 34,08 & 26,75 & 25,92 & 25,21 \\
\hline Farelo de trigo & 35,20 & 5,28 & 0,08 & - \\
\hline Calcário calcítico & 1,32 & - & 0,67 & 0,84 \\
\hline Fosfato bicálcico & - & - & 0,51 & - \\
\hline Ureia & - & - & - & 0,36 \\
\hline Sal mineral ${ }^{1}$ & 1,20 & 1,50 & 2,30 & 1,54 \\
\hline
\end{tabular}

${ }^{1}$ Composição: Cálcio 140 g; fósforo 65 g; sódio 148 $\mathrm{g}$; magnésio $5 \mathrm{~g}$; enxofre $12 \mathrm{~g}$; cobalto $107 \mathrm{mg}$; cobre $1550 \mathrm{mg}$; iodo $150 \mathrm{mg}$; manganês $1400 \mathrm{mg}$; níquel $30 \mathrm{mg}$; selênio $18 \mathrm{mg}$; zinco $4500 \mathrm{mg}$; flúor (máximo) $650 \mathrm{mg}$. simulado foram obtidas através do consumo observado dos animais experimentais conforme Johnson (1978), identificando-se o tipo de material consumido e coletando-se uma amostra semelhante ao alimento ingerido.

Para estimar a produção fecal, utilizouse o óxido de cromo como indicador externo, do $20^{\circ}$ ao $32^{\circ}$ dia de cada período, fornecido diariamente às $09 \mathrm{~h}$ em dose única de $10 \mathrm{~g}$ acondicionada em papelote durante 12 dias, com sete dias para adaptação e regulação do fluxo de excreção do marcador e cinco dias para coleta das fezes.

As fezes foram coletadas uma vez ao dia, no próprio piquete durante cinco dias, sempre após o consumo de concentrado. Houve a acuidade na coleta de fezes no piquete para não ocorrer contaminação por corpos estranhos. Posteriormente, foram armazenadas em câmara fria a $-10^{\circ} \mathrm{C}$. As amostras de fezes foram analisadas por espectrofotometria de absorção atômica (EAA) para dosagem de cromo, conforme Williams et al. (1962). A determinação da produção fecal foi realizada conforme a equação proposta por Smith e Reid (1955):

$$
\mathrm{PF}=\mathrm{OF} / \mathrm{COF}
$$

em que:

$\mathrm{PF}=$ produção fecal diária (g/dia);

$\mathrm{OF}=$ óxido de cromo fornecido (g/dia);

$\mathrm{COF}=$ concentração de óxido de cromo nas fezes (g/g MS).

Do $20^{\circ}$ ao $31^{\circ}$ dia de cada período, foi determinado a fração digestível, utilizando a fibra em detergente ácido indigestível (FDAi) (Casali et al., 2008) como indicador interno. As amostras da forragem, das fezes e dos concentrados foram incubadas no rúmen de quatro animais fistulados por 264 h (Casali et al., 2008), tendo o resíduo sido assumido como indigestível.

O consumo de MS foi obtido por meio da seguinte equação:

$\mathrm{CMS}=\left\{\left[\left(\mathrm{PF}^{*} \mathrm{CIFZ}\right)-\mathrm{IS}\right] / \mathrm{CIFR}\right\}+\mathrm{CMSS}$ 


\section{SANTANA JÚNIOR ETAL.}

em que:

$\mathrm{CMS}=$ consumo de MS (kg/dia);

$\mathrm{PF}=$ produção fecal $(\mathrm{kg} / \mathrm{dia})$;

$\mathrm{CIFZ}=$ concentração do indicador presente nas fezes $(\mathrm{kg} / \mathrm{kg})$;

$I S=$ indicador presente no suplemento $(\mathrm{kg} / \mathrm{dia})$;

$\mathrm{CIFR}=$ concentração do indicador presente na forragem $(\mathrm{kg} / \mathrm{kg})$;

CMSS= consumo de MS do suplemento $(\mathrm{kg} / \mathrm{dia})$.

Os teores de MS, matéria orgânica (MO), proteína bruta $(\mathrm{PB})$, extrato etéreo $(\mathrm{EE})$, fibra em detergente neutro (FDN) e fibra em detergente ácido (FDA) foram obtidas conforme metodologias descritas por Silva e Queiroz (2002). O teor de FDN corrigido para cinzas e proteínas foi realizado segundo recomendações de Mertens (2002). Os carboidratos totais (CHOT) foram obtidos por intermédio da equação (Sniffen et al., 1992):

$$
100-(\% \mathrm{~PB}+\% \mathrm{EE}+\% \text { cinza })
$$

enquanto os carboidratos não-fibrosos (CNF), pela diferença entre CHOT e FDNcp. Os teores de nutrientes digestíveis totais (NDT) foram calculados segundo Weiss (1999), mas utilizando a FDN e os CNF corrigidos para cinza e proteína, pela seguinte equação:

$\mathrm{NDT}(\%)=\mathrm{PBD}+\mathrm{FDNcpD}+\mathrm{CNFD}+2,25 \mathrm{EED}$

em que:

$\mathrm{PBD}=\mathrm{PB}$ digestível;

$\mathrm{FDNcpD}=\mathrm{FDNcp}$ digestível;

CNFD= CNF digestíveis;

$\mathrm{EED}=\mathrm{EE}$ digestível.

A avaliação do comportamento foi realizada no $28^{\circ}$ dia de cada período, totalizando então quatro avaliações, sendo feitas observações a cada 10 minutos conforme metodologia de Silva et al. (2008), por um período de 24 horas, a fim de identificar o tempo destinado ao pastejo, ruminação, alimentação no cocho e outras atividades. Os animais foram avaliados visualmente, por dois observadores treinados para cada tratamento, sendo os mesmos, posicionados estrategicamente de forma a não incomodar os animais. Para saber o tempo gasto em cada atividade foram utilizados relógios digitais. Para o período noturno, não houve a necessidade de iluminação artificial, pois o período de coleta coincidiu com a fase de lua cheia em todas as avaliações ( 56 em 56 dias que se assemelha com 02 ciclos da lua).

As variáveis comportamentais estudadas foram: tempo de pastejo (PAS), tempo de ruminação (RUM), tempo de alimentação no cocho (COC) e tempo em outras atividades (OUT). As atividades comportamentais foram consideradas mutuamente excludentes, conforme definição de Pardo et al. (2003).

O tempo gasto pelos animais na seleção e apreensão da forragem, incluindo os curtos espaços de tempo utilizados no deslocamento para a seleção da forragem foi considerado tempo de pastejo (Hancock, 1953). O tempo de ruminação correspondeu aos processos de regurgitação, remastigação, reinsalivação e redeglutição. O tempo de alimentação no cocho foi o tempo despendido pelo animal no consumo de suplemento. Enquanto o tempo em outras atividades (descanso, consumo de água, interações, etc.), foram todas as atividades com exceção das citadas acima.

O tempo de alimentação total (TAT) e de mastigação total (TMT) foi determinado pelas equações abaixo:

$$
T A T=P A S+C O C
$$

em que:

$\mathrm{PAS}=$ tempo de pastejo em minutos; $\mathrm{COC}=$ tempo de alimentação no cocho em minutos.

$T M T=P A S+R U M+C O C$

em que:

PAS= tempo de pastejo em minutos;

$\mathrm{RUM}=$ tempo de ruminação em minutos;

$\mathrm{COC}=$ tempo de alimentação no cocho em minutos. 


\section{CORRELAÇÕES ENTRE DIGESTIBILIDADE E COMPORTAMENTO}

A discretização das séries temporais foi realizada diretamente nas planilhas de coleta de dados, com a contagem dos períodos discretos de pastejo, ruminação, outras atividades e alimentação no cocho, conforme descrito por Silva et al. (2006). A duração média de cada um dos períodos discretos foi obtida pela divisão dos tempos diários de cada uma das atividades pelo número de períodos discretos da mesma atividade.

Foram realizadas observações por dois períodos do dia (manhã e tarde), com três repetições por período (Burger et al., 2000), a fim de determinar o número de mastigações merícicas por bolo ruminado (MMB) e o tempo gasto para ruminação de cada bolo (TBo).

As variáveis, número de bolo ruminado por dia (BOL), velocidade de mastigação (VeM), tempo por mastigação merícica (TeM) e mastigações merícicas por dia (MMnd) foram calculadas pelas equações abaixo:

$\mathrm{BOL}=\mathrm{RUM} / \mathrm{TBO}$

$\mathrm{VeM}=\mathrm{MMB} / \mathrm{TBO}$

em que:

$\mathrm{VeM}=$ mastigações merícicas;

$\mathrm{MMB}=$ número de mastigações merícicas por bolo; $\mathrm{TBo}=$ tempo por bolo ruminado em segundos.

$\mathrm{TeM}=\mathrm{TB} / \mathrm{MMB}$

em que:

$\mathrm{TeM}=$ tempo por mastigação merícica em segundos;

$\mathrm{TBo}=$ tempo por bolo ruminado em segundos; $\mathrm{MMB}=$ número de mastigações merícicas por bolo .

$\mathrm{MMnd}=\mathrm{BOL}{ }^{*} \mathrm{MMB}$

em que:

MMnd= número de mastigações merícicas por dia; $\mathrm{BOL}=$ número de bolos ruminados por dia; $\mathrm{MMB}=$ número de mastigações merícicas por bolo .

Durante os mesmos períodos de avaliação do comportamento animal, quando os animais estavam em atividade de pastejo a mais de 30 minutos, foi registrada a taxa de bocado (TxB) dos animais de cada tratamento, estimada por meio do tempo gasto pelo animal para realizar 20 bocados (Hodgson, 1982). Para o cálculo da massa de bocado (MaB), dividiu-se o consumo diário pelo total de bocados diários (Jamieson e Hodgson, 1979).

Também foi coletado o número de bocados e o tempo desprendido entre deglutições. Os resultados das observações de bocados e deglutição foram registrados em seis ocasiões durante o dia, conforme Baggio et al. (2009), sendo três avaliações durante a manhã e três à tarde, e usados também para determinar o número de bocados por dia (NBD), que é o produto entre taxa de bocado e tempo de pastejo.

As correlações foram feitas por meio de análise de correlações lineares de Pearson e teste ' $t$ ', e processadas pelo Programa SAEGSistema de Análises Estatísticas e Genéticas, sendo consideradas significativas quando $\mathrm{p}<0,05$. Foram utilizados os seguintes parâmetros:

- Digestibilidade: coeficientes de digestibilidade da MS (CDMS), matéria orgânica (CDMO), fibra em detergente neutro (CDFDN), carboidratos não-fibrosos (CDCNF), carboidratos totais (CDCHOT), proteína bruta(CDPB), extrato etéreo (CDEE) e os nutrientes digestíveis totais (NDT);

- Comportamento ingestivo: tempo de pastejo (PAS), tempo de ruminação (RUM), tempo de alimentação no cocho (COC), tempo em outras atividade (OUT), tempo de alimentação total (TAT), tempo de mastigação total (TMT), número de períodos de pastejo (NPP), número de períodos de ruminação (NPR), número de períodos de alimentação no cocho (NPC) e número de períodos de outras atividades (NPO), tempo por período de pastejo (TPP), tempo por período de ruminação (TPR), tempo por período de alimentação no cocho (TPC) e tempo por período de outras atividades (TPO), taxa de bocado (TxB), massa de boca- 


\section{SANTANA JÚNIOR ETAL.}

do (MaB), número de bocados entre deglutições (BDe), tempo entre deglutições (TDe), número de bocado por dia (NBD), número de mastigações merícicas por bolo (MMB), tempo por bolo ruminado (TBo), velocidade de mastigação (VeM), tempo por mastigação (TeM), número de mastigações merícicas por dia (MMnd), número de bolos ruminados por dia (BOL).

\section{RESULTADOSEDISCUSSÃO}

Algumas variáveis não se encontram expostas nas tabelas devido a significância superior ao nível considerado $(\mathrm{p}<0,05)$.

Verificou-se uma correlação positiva entre o tempo de pastejo e o CDFDN (tabela II). Essa correlação do CDFDN com o pastejo ocorreu provavelmente devido a taxa de passagem do volumoso, onde o limitante é o efeito de enchimento provocado pela FDN em animais em pastagens tropicais, pois quanto maior a digestibilidade da FDN, menor tempo a mesma ficará no rúmen, pro- piciando assim, maior oportunidade de pastejo do animal. Esse efeito foi citado por Berchielli et al. (2006), que aborda que as limitações físicas estão relacionadas com a degradação do alimento e com o fluxo da digesta pelo rúmen e outras partes do aparelho gastrointestinal. Allen (2000) afirmou que para cada aumento de uma unidade percentual de CDFDN, acarretaria em um incremento de $170 \mathrm{~g}$ de ingestão de MS.

Correlações negativas foram verificadas entre o tempo de OUT e CDFDN. Como as atividades comportamentais são mutuamente excludentes, para a realização de outras atividades, o animal não pode estar pastejando, portanto para um maior consumo de forragem é necessário a elevação do tempo de pastejo.

O COC apresentou correlações negativas com CDMS, CDMO, CDCNF, CDCHOT. Observa-se uma correlação positiva entre o TAT e TMT com o CDFDN, sendo o mesmo explicado pelo motivo de que para maiores

Tabela II. Correlações lineares entre o comportamento ingestivo e as digestibilidades em novilhas mestiças a pasto. (Linear correlations among ingestive behavior and digestibility in grazing crossbred heifers).

\begin{tabular}{lcccccc}
\hline & PAS & RUM & OUT & COC & TAT & TMT \\
\hline CDMS & - & - & - & $-0,65(0,0388)$ & - & - \\
CDMO & - & - & - & $-0,65(0,0410)$ & - & - \\
CDFDN & $0,70(0,0264)$ & - & $-0,65(0,0161)$ & - & $0,73(0,0199)$ & $0,75(0,0161)$ \\
CDCNF & - & - & - & $-0,64(0,0425)$ & - & - \\
CDCHOT & - & - & - & $-0,65(0,0407)$ & - & - \\
CDPB & - & - & - & - & - & - \\
CDEE & - & - & - & - & - & - \\
NDT & - & - & - & - & - & - \\
\hline
\end{tabular}

$\mathrm{PAS}=$ tempo de pastejo; RUM= tempo de ruminação; OUT= tempo de outras atividades; $C O C=$ tempo de alimentação no cocho; TAT= tempo de alimentação total; TMT= tempo de mastigação total; CDMS= coeficiente de digestibilidade da matéria seca; $\mathrm{CDMO}=$ coeficiente de digestibilidade de matéria orgânica; CDFDN= coeficiente de digestibilidade de fibra em detergente neutro; $\mathrm{CDCNF}=$ coeficiente de digestibilidade de carboidratos não-fibrosos; $\mathrm{CDCHOT}=$ coeficientes de digestibilidade de carboidratos totais; $\mathrm{CDPB}=$ coeficiente de digestibilidade de proteína bruta; $\mathrm{CDEE}=$ coeficiente de digestibilidade de extrato etéreo; $\mathrm{NDT}=$ nutrientes digestíveis totais

Os valores de significância $(p<0,05)$ estão apresentados entre os parênteses. 


\section{CORRELAÇÕES ENTRE DIGESTIBILIDADE E COMPORTAMENTO}

tempos de alimentação e mastigação total são necessários maiores digestibilidades da FDN. Uma maior mastigação, provavelmente é decorrente da elevação do conteúdo fibroso da dieta, com isso o animal consumirá mais kg de MS diária por kg de ganho de peso.

O NPP e o NPR apresentaram correlações negativas com CDMS, CDMO, CDCNF, CDCHOT, NDT $(r=0,93)$, CDPB (tabela III). Também, verificou-se correlação acima de 0,90 para o NDT, podendo também ser incluída em equações de predição de consumo.

O NPO apresentou correlação positiva com o CDEE. Enquanto as correlações com as variáveis do consumo seguem a mesma discussão realizada para o OUT. Correlações negativas foram encontradas entre o NPO e CDMS, CDMO, CDCNF, CDCHOT, NDT e CDPB. Quanto menor é o número de períodos de outras atividades, maior é a tendência ao consumo, e consequentemente à digestibilidade.

ONPC correlacionou negativamente com CDMO, CDPB. As justificativas das correlações positivas são em virtude do maior NPC estar associado ao maior fornecimento de suplemento, e o mesmo apresentarem maior teor de NDT e PB.

O TPP apresentou correlações inversamente proporcionais ao NPP, pois a elevação de uma variável provoca a diminuição da outra variável. O TPR correlacionou positi-

Tabela III. Correlações lineares entre os períodos do comportamento ingestivo e as digestibilidades em novilhas mestiças a pasto. (Linear correlations among periods of ingestive behavior and digestibility in grazing crossbred heifers).

\begin{tabular}{lcccccccc}
\hline & NPP & NPR & NPO & NPC & TPP & TPR & TPO & TPC \\
\hline CDMS & $-0,89$ & $-0,85$ & $-0,90$ & - & 0,97 & - & - & - \\
& $(0,0014)$ & $(0,0039)$ & $(0,0012)$ & & $(0,0000)$ & & & \\
CDMO & $-0,88$ & $-0,83$ & $-0,88$ & $-0,63$ & 0,97 & 0,92 & - & - \\
& $(0,0020)$ & $(0,0050)$ & $(0,0019)$ & $(0,0468)$ & $(0,0000)$ & $(0,0007)$ & & \\
CDFDN & & & & & & & & \\
CDCNF & $-0,88$ & $-0,85$ & $-0,90$ & - & 0,88 & 0,81 & 0,66 & - \\
& $(0,0020)$ & $(0,0038)$ & $(0,0010)$ & & $(0,0017)$ & $(0,0068)$ & $(0,0360)$ & - \\
CDCHOT & $-0,88$ & $-0,85$ & $-0,90$ & - & 0,95 & 0,89 & - & - \\
CDPB & $(0,0017)$ & $(0,0040)$ & $(0,0012)$ & & $(0,0001)$ & $(0,0014)$ & & \\
& $-0,81$ & $-0,74$ & $-0,77$ & $-0,72$ & 0,88 & 0,88 & - & - \\
CDEE & $(0,0073)$ & $(0,0186)$ & $(0,0126)$ & $(0,0216)$ & $(0,0021)$ & $(0,0018)$ & & \\
& - & - & 0,68 & - & - & - & $-0,64$ & - \\
NDT & $-0,93$ & $-0,92$ & $-0,96$ & - & 0,94 & 0,87 & $0,0423)$ & \\
& $(0,0005)$ & $(0,0005)$ & $(0,0001)$ & & $(0,0002)$ & $(0,0022)$ & $(0,0193)$ & \\
\hline
\end{tabular}

$\mathrm{NNP}=$ número de períodos de pastejo; $\mathrm{NPR}=$ número de períodos de ruminação; NPO= número de períodos de outras atividades; NPC= número de períodos de alimentação no cocho; TPP= tempo por período de pastejo; TPR= tempo por período de ruminação; TPO= tempo por período de outras atividades; TPC= tempo por período de alimentação no cocho; $C D M S=$ coeficiente de digestibilidade da MS; CDMO=coeficiente de digestibilidade da matéria orgânica; $C D C N F=$ coeficiente de digestibilidade de carboidratos não fibrosos; CDCHOT = coeficiente de digestibilidade de carboidratos totais; NDT= nutrientes digestíveis totais; $\mathrm{CDEE}=$ coeficiente de digestibilidade de extrato etéreo; CDPB= coeficiente de digestibilidade de proteína bruta.

Os valores de significância $(p<0,05)$ estão apresentados entre os parênteses. 


\section{SANTANA JÚNIOR ETAL.}

vamente com CDMO, CDCNF, CDCHOT, NDT e CDPB. O TPO correlacionou positivamente com CDCNF e NDT. E negativamente com CDEE. Para maiores tempos por período de outras atividades é necessário uma elevação das digestibilidades dos CNF e NDT, tudo isso visando atender as exigências para mantença e ganho diário esperado.

Correlações negativas foram verificadas entre a TxB e CDMS, CDMO, CDCNF, CDCHOT e CDPB (tabela IV). Um maior consumo só é obtido quando de consegue elevar a taxa de passagem da alimentação, porém a máxima taxa de passagem que pode ser alcançada $(8 \% / \mathrm{h})$, provoca diminuição das digestibilidades. Berchielli et al. (2006) cita que o aumento no nível de ingestão para duas vezes a necessidade de manutenção, resulta em 1 a 2 pontos percentuais de redução na digestibilidade da MS do alimento no ruminante.

OTDe correlacionou positivamente com CDMS, CDMO, CDCNF, CDCHOT, NDT, CDPB. O tempo por deglutição é o tempo gasto pelo animal até alcançar o volume desejado para realizar a deglutição, então quanto maior o tempo por deglutição, maior está sendo as digestibilidades, devido ao maior tempo de alimentando no trato gastrointestinal (TGI). Inversamente proporcional, o consumo elevado só é atingido com diminuição do tempo por deglutição o que acarreta maior velocidade de ingestão.

Verificou-se correlação negativa entre o NDB e NDT. A MaB e BDe não apresentaram correlações com nenhuma das variáveis da digestibilidade. Entretanto, Stobbs (1973) e Chaccon e Stobbs (1976) em seus trabalhos pioneiros com plantas forrageiras tropicais revelaram a importância da massa do bocado relativamente aos demais componentes do comportamento ingestivo. Hodgson (1985) e Bremm et al. (2008) confirmaram também, afirmando que a massa do bocado é a variável mais importante na determinação do consumo de animais em pastejo.

O número de mastigações merícicas por bolo ruminal apresentou correlações nega-

Tabela IV. Correlações lineares entre os aspectos do comportamento ingestivo (bocado e deglutição) e as digestibilidades em novilhas mestiças a pasto. (Linear correlations between aspects of intake behavior (bit and swallowing) and digestibility in grazing crossbred heifers).

\begin{tabular}{lccccc}
\hline & TxB & MaB & BDe & TDe & NBD \\
\hline CDMS & $-0,73(0,0206)$ & - & - & $0,90(0,0010)$ & - \\
CDMO & $-0,73(0,0204)$ & - & - & $0,91(0,0008)$ & - \\
CDFDN & - & - & - & - & - \\
CDCNF & $-0,66(0,0385)$ & - & - & $0,82(0,0058)$ & - \\
CDCHOT & $-0,71(0,0239)$ & - & - & $0,90(0,0012)$ & - \\
CDPB & $-0,69(0,0289)$ & - & - & $0,76(0,0149)$ & - \\
CEE & - & - & - & - & - \\
NDT & - & - & - & $0,76(0,0136)$ & $-0,76(0,0135)$
\end{tabular}

$\mathrm{TxB}=$ taxa de bocados; $\mathrm{MaB}=$ massa do bocado; $\mathrm{BDe}=$ bocados por deglutição; $\mathrm{TDe}=$ tempo por deglutição; $\mathrm{NBD}=$ número de bocados por dia; $\mathrm{CMST}=$ consumo de MS total; CDMS= coeficiente de digestibilidade da $\mathrm{MS} ; \mathrm{CDMO}=$ coeficiente de digestibilidade de matéria orgânica; $\mathrm{CDFDN}=$ coeficiente de digestibilidade de fibra em detergente neutro; $\mathrm{CDCNF}=$ coeficiente de digestibilidade de carboidratos não fibrosos; $C D C H O T=$ coeficiente de digestibilidade de carboidratos totais; $\mathrm{CDPB}=$ coeficiente de digestibilidade de proteína bruta; $\mathrm{CDEE}=$ coeficiente de digestibilidade de extrato etéreo; $N D T=$ nutrientes digestíveis totais.

Os valores de significância $(p<0,05)$ estão apresentados entre os parênteses.

Archivos de zootecnia vol. 61, núm. 236, p. 556. 


\section{CORRELAÇÕES ENTRE DIGESTIBILIDADE E COMPORTAMENTO}

tivas $(-0,63)$ com NDT. Observa-se que para um menor teor de NDT é oriundo da elevação do consumo de forragem, provocando um maior número de mastigação realizada no durante o dia. Salienta-se que existe uma associação negativa entre o suplemento e as atividades dos aspectos da ruminação do comportamento ingestivo (Missio et al., 2010).

\section{BIBLIOGRAFIA}

Allen, M.S. 2000. Effects of diet on short-term regulation of feed intake by lactating dairy cattle. J Dairy Sci, 83: 1598-1624.

Baggio, C., Carvalho, P.C.F., Silva, J.L.S., Anghinoni, I., Lopes, M.L.T. e Thurow, J.M. 2009. Padrões de deslocamento e captura de forragem por novilhos em pastagem de azevémanual e aveia-preta manejada sob diferentes alturas em sistema de integração lavourapecuária. Rev Bras Zootecn, 38: 215-222.

Berchielli, T.T., Pires, A.V. e Oliveira, S.G. 2006. Nutrição de ruminantes. In: Berchielli, T.T.; Pires, A.V.; Oliveira, S.G. (Eds.). $1^{\underline{a}}$ ed. FUNEP. Jaboticabal. pp. 583.

Bremm, C., Rocha, M.G., Freitas, F.K., Macari, S., Elejalde, D.A.J. e Roso, D. 2008. Comportamento ingestivo de novilhas de corte submetidas a estratégias de suplementação em pastagens de aveia e azevém. Rev Bras Zootecn, 37: 1161-1167.

Burger, P.J., Pereira, J.C., Queiroz, A.C., Silva, J.F.C., Valadares Filho, S.C., Cecon, P.R. e Casali, A.D.P. 2000. Comportamento ingestivo de bezerros holandeses alimentados com dietas contendo diferentes níveis de concentrado. Rev Bras Zootecn, 29: 236-242.

Cardoso, E., Carvalho, S., Galvani, D.B., Pires, C.C. e Gasperin, B.G. 2006. Comportamento ingestivo de cordeiros alimentados com dietas contendo diferentes níveis de fibra em detergente neutro. Ciênc Rural, 36: 604-609.

Casali, A.O., Detmann, E., Valadares Filho, S.C., Pereira, J.C., Henriques, L.T., Freitas, S.G. e Paulino, M.F. 2008. Influência do tempo de incubação e do tamanho de partículas sobre os teores de compostos indigestíveis em alimentos e fezes bovinas obtidos por procedimentos in

\section{CONCLUSÃO}

O tempo de pastejo e ruminação não apresentaram relação com os demais coeficientes de digestibilidade aparente de novilhas mestiças a pasto.

O coeficiente da digestibilidade da fibra em detergente neutro provoca variações no tempo de pastejo.

situ. Rev Bras Zootecn, 37: 335-342.

Chaccon, E.A. and Stobbs, T.H. 1976. Influence of progressive defoliation of a grass sward on the eating behaviour of cattle. Aust J Agric Res, 27: 709-727.

Hancock, J. 1953. Grazing behaviour of cattle. Animal Breeding Abstract, 21: 1-13.

Hodgson, J. 1982. Ingestive behavior. In: Leaver, J.D. (Ed.). Herbage intake handbook. British Grassland Society. Hurley. pp. 113.

Hodgson, J. 1985. The control of herbage intake in the grazing ruminant. $P$ Nutr Soc, 44: 339-346. Jamieson, W.S. and Hodgson, J. 1979. The effect of variation in sward characteristics upon the ingestive behavior and herbage intake of calves and lambs under continuous stocking management. Grass Forage Sci, 34: 273-281. Johnson, A.D. 1978. Sample preparation and chemical analysis of vegetation. In: Manetje, L.T. (Ed.). Measurement of grassland vegetation and animal production. Commonwealth Agricultural Bureau. Aberystwyth. pp. 96-102.

Mertens, D.R. 2002. Gravimetric determination of amylase-treated neutral detergent fiber in feeds with refluxing in beakers or crucibles: collaborative study. J AOAC Int, 85: 1217-1240.

Minson, D.G. 1990. Forage in ruminant nutrition. Academic Press. New York. pp. 483.

Missio, R.L., Brondani, I.L., Alves Filho, D.C., Silveira, M.F., Freitas, L.S. e Restle, J. 2010. Comportamento ingestivo de tourinhos terminados em confinamento, alimentados com diferentes níveis de concentrado na dieta. Rev Bras Zootecn, 39: 1571-1578.

Pardo, R.M.P., Fischer, V., Balbinotti, M., Zanela, M.B., Moreno, C.B., Ferreira, E.X., Vinhas, R.I. e Monks, P.L. 2003. Comportamento ingestivo 


\section{SANTANA JÚNIOR ETAL.}

diurno de novilhos em pastejo a níveis crescentes de suplementação energética. Rev Bras Zootecn, 32: 1408-1418.

Silva, D.J. e Queiroz, A.C. 2002. Análise de alimentos (métodos químicos e biológicos). Universidade Federal de Viçosa. Viçosa. pp. 235.

Silva, J.F.C. e Leão, M.I. 1979. Fundamentos de nutrição dos ruminantes. Livroceres. Piracicaba. $380 \mathrm{pp}$.

Silva, R.R., Silva, F.F., Prado, I.N., Carvalho, G.G.P., Franco, I.L., Almeida, V.S., Cardoso, C.P. e Ribeiro, M.H.S. 2006. Comportamento ingestivo de bovinos. Aspectos metodológicos. Arch Zootec, 55: 293-296.

Silva, R.R., Prado, I.N., Carvalho, G.G.P., Santana Junior, H.A., Silva, F.F. e Dias, D.L.S. 2008. Efeito da utilização de três intervalos de observações sobre a precisão dos resultados obtidos no estudo do comportamento ingestivo de vacas leiteiras em pastejo. Ciênc Anim Bras, 9: 319-326.

Smith, A.M. and Reid, J.T. 1955. Use of chromic oxide as an indicator of fecal output for the purpose of determining the intake of a pasture herbage by grazing cows. J Dairy Sci, 38: 515524.

Sniffen, C.J., O'connor, J.D., Van Soest, P.J., Fox, D.G. and Russell. J.B. 1992. A net carbohydrate and protein system for evaluating cattle diets: IICarbohydrate and protein availability. J Dairy Sci, 70: 3562-3577.

Stobbs, T.H. 1973. The effect of plant structure on the intake of tropical pastures. I. Variation in the bite size of grazing cattle. Aust J Agr Res, 24: 809-819.

Van Soest, P.J. 1994. Nutritional ecology of the ruminants. $2^{a}$ ed. Cornell University. Ithaca. pp. 476.

Weiss, W.P. 1999. Energy prediction equations for ruminant feeds. In: Cornell Nutrition Conference for Feed Manufacturers, 61. Proceedings... Cornell University. Ithaca. pp. 176-185.

Willians, C.H., David, D.J. and Lilma, O. 1962. The determination of cromic oxide in faeces samples by atomic absorption spectrophotometry. $J$ Agr Sci, 59: 381-385. 